\title{
Upper Eyelid Pseudocyst Related to Forehead Filler Migration: A Rare Complication of an Illegal Filler Injection
}

\author{
Da Woon Lee ${ }^{1}$, Eun Soo Park ${ }^{2}$, \\ Wang Seok Lee ${ }^{1}$, Min Sung Tak ${ }^{1}$, \\ Ah Rim Moon ${ }^{3}$

\begin{abstract}
${ }^{1}$ Department of Plastic and Reconstructive Surgery, Soonchunhyang Seoul Hospital, Soonchunhyang University College of Medicine, Seoul; ${ }^{2}$ Department of Plastic and Reconstructive Surgery, Soonchunhyang Bucheon Hospital, Soonchunhyang ${ }^{3}$ Department of Pathology, College of Medicine, Soonchunhyang Bucheon Hospital, Soonchunhyang University College of Medicine, Bucheon, Korea
\end{abstract} \\ University College of Medicine, Bucheon;
}

This work was supported by the Soonchunhyang University Research Fund.

No potential conflict of interest relevant to this article was reported.

\begin{abstract}
We report a very rare case of unilateral blepharoptosis and swelling as an unusual complication of a filler injection. The patient received a filler injection into the forehead 4 years previously by an unlicensed practitioner. In the operation, an encapsulated yellowish cyst with inflammation was found to be adhered to the orbital septum and was excised. To prevent additional inferior migration of the remaining foreign body in the forehead, the retro-orbicularis fascia and preaponeurotic fat pad area were sutured, with the exception of the levator aponeurosis. This cyst-like mass was histopathologically proven to be a multiple pseudocyst. After excision, the swelling disappeared and the ptotic eyelid also improved. The galea is connected with the posterior orbicularis fascia. The galea and posterior orbicularis fascia layer can function as a pathway through which the injected material can migrate from the forehead to the upper eyelid. Weakening of the orbicularis retaining ligament and leakage of the foreign body through the supraorbital foramen may also cause filler migration. This case underscores the need for clinicians to be aware of the potential migration of filler even many years after an injection. We advise that filler injections should be performed by trained physicians and that it should be made known that migration is possible.
\end{abstract}

Keywords Blepharoptosis, Dermal filler, Eyelids

\section{INTRODUCTION}

In the past few decades, facial fillers have been recognized as ideal for use as injectable therapeutic tools for soft tissue augmentation and rejuvenation. With recognition of its value, filler injection has become one of the most commonly performed cosmetic procedures. Clinically used dermal fillers are composed of silicone parti-

Received: Apr 5, 2017 Revised: Apr 26, 2017 Accepted: Apr 27, 2017 Correspondence: Eun Soo Park Department of Plastic and Reconstructive Surgery, Soonchunhyang Bucheon Hospital, Soonchunhyang University College of Medicine, 170 Jomaru-ro, Wonmi-gu, Bucheon 14584, Korea. E-mail: peunsoo@schmc.ac.kr

Copyright () 2017 The Korean Society for Aesthetic Plastic Surgery.

This is an Open Access article distributed under the terms of the Creative Commons Attribution Non-Commercial License (http://creativecommons.org/licenses/by-nc/4.0/) which permits unrestricted non-commercial use, distribution, and reproduction in any medium, provided the original work is properly cited. $\quad w w w . e-a a p s . o r g$ cles, autologous fat, bovine collagen, paraffin, and polytetrafluoroethylene [1]. Fillers should have the properties of biocompatibility, safety, and stability at the implant site and the ability to maintain their volume, remain pliable, and induce minimal foreign body reactions, without causing foreign body granuloma [2]. However, fillers are not autologous tissue; instead, they are essentially foreign bodies that can cause an unwanted inflammatory response in individuals. Additionally, fillers are injected in a blind procedure in which the practitioner is unable to note exactly where the material is being directed. Due to these problems, fillers can cause potential complications. In East Asian countries, the illegal injection of various substances by unlicensed practitioners has been extensively carried out on various parts of the body, including the face [3]. This dramatically increases the risk of complications. We herein report a very rare case of filler migration to the eyelid, causing unilateral blepharoptosis and swelling. 


\section{CASE}

Patient

A 65-year-old male complained of swelling and blepharoptosis of his right upper eyelid. His right upper eyelid was swollen overall and ptotic. Severe blepharoptosis (marginal reflex distance, $1 \mathrm{~mm}$ ) of the right upper lid was observed (Fig. 1). The cystic mass did not affect visual acuity or limit ocular motility. The patient had no medical comorbidities and no history of trauma in the periorbital

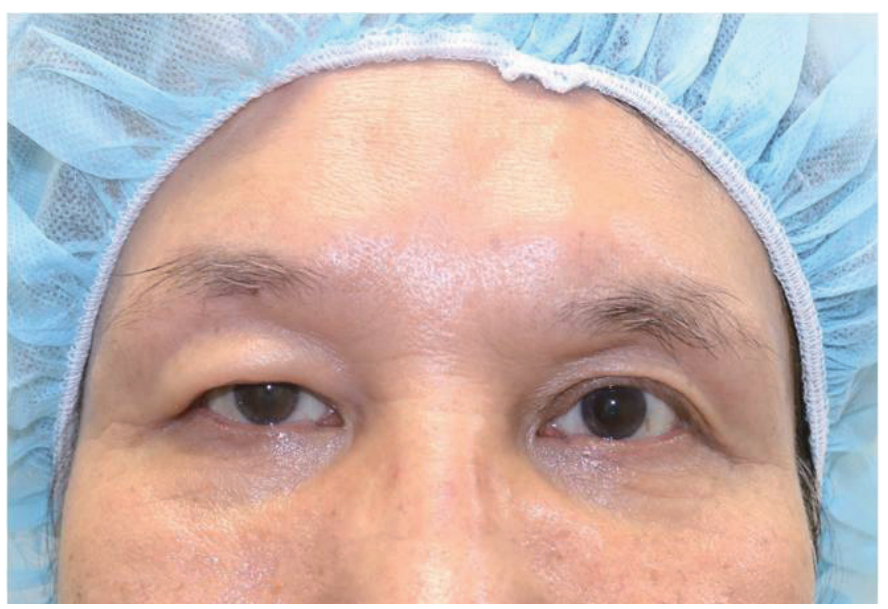

Fig. 1. A 65-year-old male presenting with swelling and blepharoptosis on the right upper eyelid that developed after an illegal filler injection to the forehead.

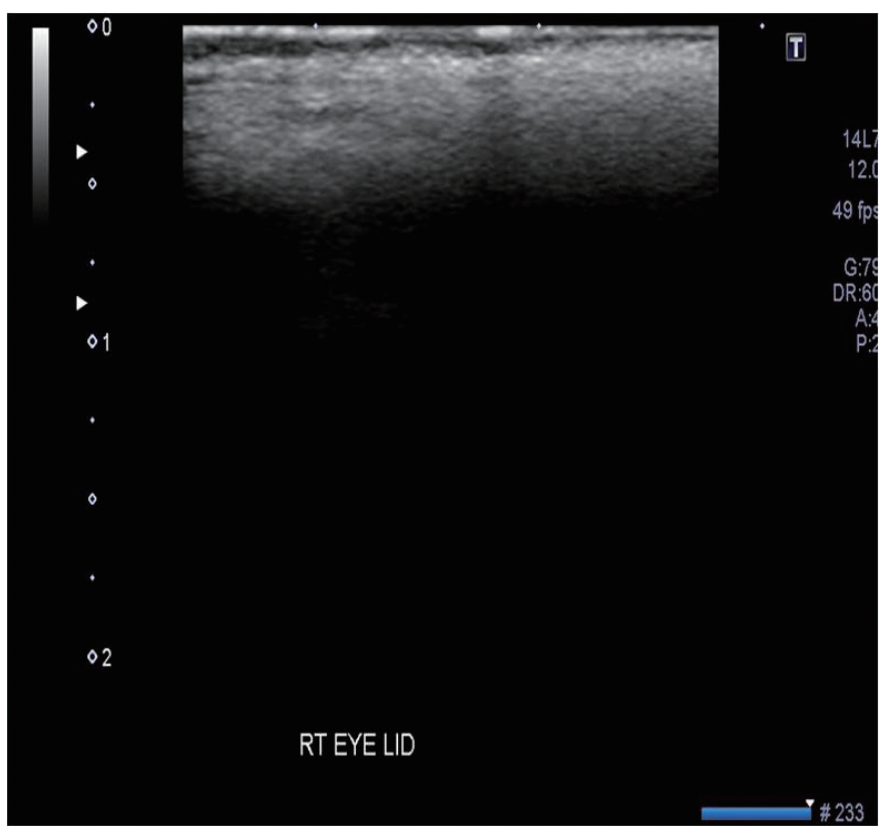

Fig. 2. On ultrasonography, there was an area of high attenuation with posterior shadowing, which was the result of chronic inflammation in the upper eyelid. region. After further questioning, the patient stated that he received an illegal filler injection in the glabellar area 4 years previously by an unlicensed practitioner. After the filler injection, the patient suffered from spontaneous painful swelling and blepharoptosis, even after undergoing foreign body removal at a local clinic several months previously. After surgery, his symptoms did not improve, and he was referred to our outpatient department. On ultrasonography, there was an area of hyperechoic attenuation with posterior shadowing on the superficial layer of the upper eyelid (Fig. 2). This was presumed to be an area of chronic inflammation due to the filler injection. Thus, we planned a surgical excision to reduce the volume of the swollen eyelid and to obtain a biopsy through a skin incision in the upper eyelid crease.

In the intraoperative field, we designed an incision line above the right upper eyelid crease where swelling was observed. After the incision was made, a foreign body accumulation in the preseptal fat was identified, and it was adhered to the orbital septum. By checking the movement of the levator through spontaneous eye opening, the foreign body mass was confirmed to be adhered to the orbital septum; moreover, it protruded forward, which interfered with the movement of the levator aponeurosis. A yellow and rubbery cyst with inflammation was observed on the orbital septum and excised (Fig. 3). The mass was fixed with thick adhesions to the surrounding structures and fibrotic tissue. Because the patient had unilateral mechanical blepharoptosis due to the foreign body, the foreign body was removed along with the preseptal fat pad and anterior sheath of orbital septum, and manipulation of the levator aponeurosis was not performed. To prevent additional inferior migration of the remaining foreign body in the forehead, the retro-orbicularis fascia and preaponeurotic fat pad area were sutured, with the exception of the levator aponeurosis, using polydioxanone No. 6-0. The operation was finished after tarsal plate fixation

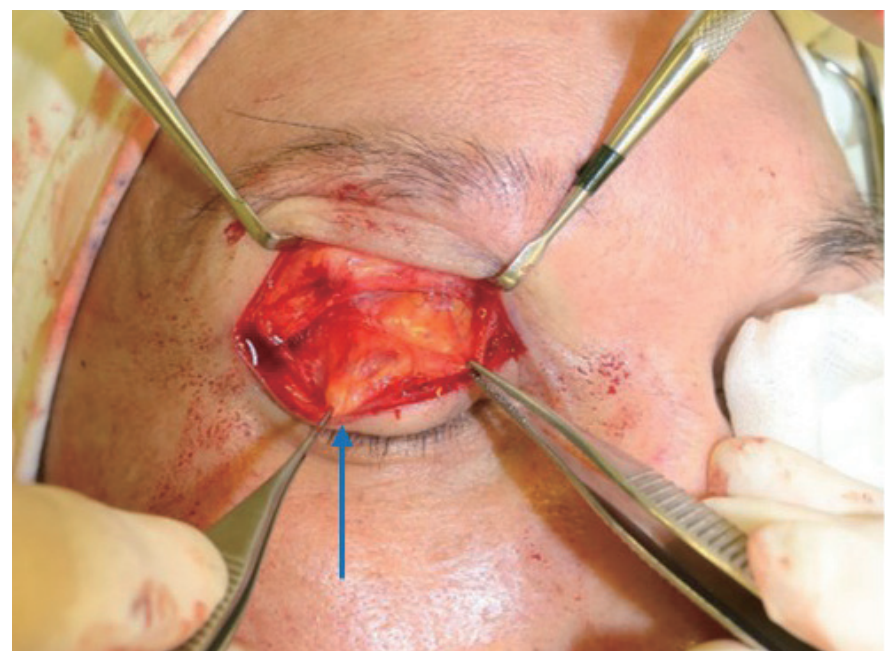

Fig. 3. The intraoperative findings included a protruding cystic mass on the orbital septum, shown with a blue arrow. 


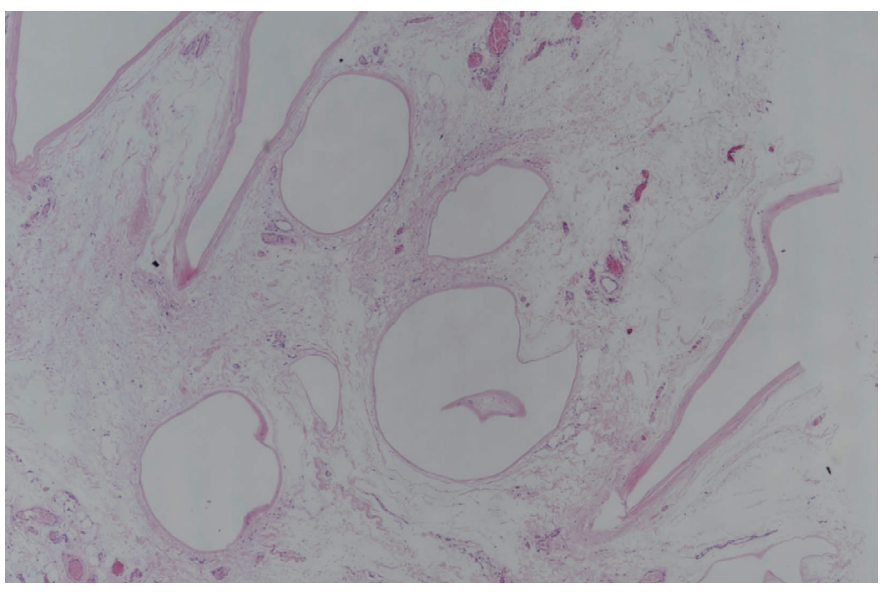

Fig. 4. The pathologic specimen was considered to be an empty pseudocyst and was thought to be removed filler, and a tissue that was presumed to be preaponeurotic fat was observed (hematoxylin and eosin stain [H \& E], original magnification $\times 40$ magnification].

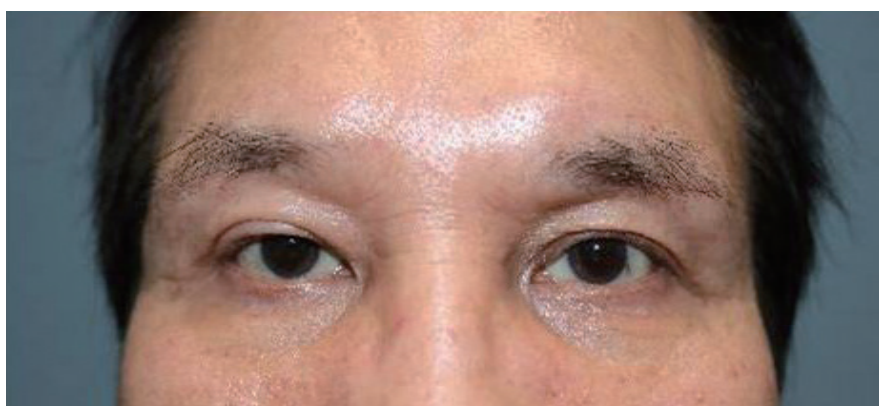

Fig. 5. Five months after surgery, there was no swelling or blepharoptosis.

was performed at 3 points (medial, central, and lateral, based on the mid-pupil line).

\section{Results}

Until 1 week after the operation, bruising and swelling were sustained. No problems were noted in terms of wound disruption and decreased visual acuity at the suture site. However, 5 months later, the swelling and the ptotic eyelid disappeared (Fig. 4). The marginal reflex distance improved objectively to more than $2 \mathrm{~mm}$. No palpable mass remained. He did not complain of other complications of blepharoplasty, such as Hering ptosis, edema, or postinflammatory hyperpigmentation. Although the patient experienced slight discomfort due to a mild scar formation reaction, he was very pleased with the results.

This mass was histopathologically proven to be several pseudocysts without inner epithelium, and is thought to have been the result of an inflammatory reaction. Based on the microscopic examination of a stained sample, the pseudocyst was thought to be a trace of the filler. Adipose cells were mostly seen around the pseudocyst

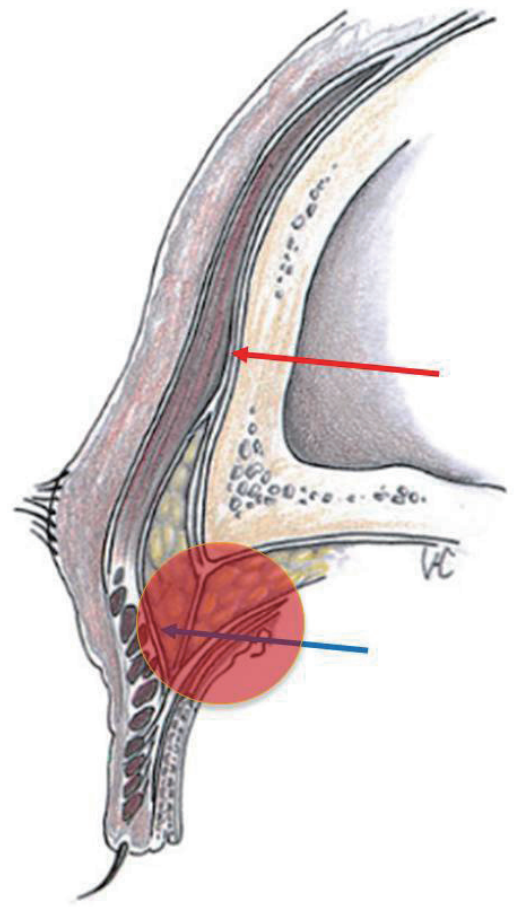

Fig. 6. The galea (red arrow) and the posterior orbicularis fascia (blue arrow), which may be potential pathways of filler migration. The roughly marked parts are preseptal fat and preaponeurotic fat at the base of the orbital septum.

and were presumed to be preaponeurotic fat (Fig. 5).

\section{DISCUSSION}

After the postoperative review, the blepharoptosis of the patient was judged to have been caused by foreign body mass adherence to the orbital septum and anterior protrusion. The foreign body accumulation with the pseudocyst of the right upper eyelid was thought to be due to migration of the unidentified filler injected into the forehead 4 years previously. We searched the literature for information on the mechanism of filler migration. Regarding the injection technique, injection of the filler with a high-volume injection or pressure may cause the filler to move over layers susceptible to overflow or weak resistance [4]. Filler migration can also be caused by gravity or dislodging of the old filler due to an additional filler injection [4]. Massage to make the filler have an even distribution, or the natural movement of the muscles such as the frontalis may also be causes. Massage was carried out in the patient described in this report for a considerable period (more than 1 week) to address the compensatory movement in the frontalis muscle caused by swelling and blepharoptosis.

Granuloma formation after facial filler injection occurs in $0.1 \%$ to $0.01 \%$ of cases, and it is most commonly caused by oily substances such as silicone or paraffin [5]. The galea, which is at the same 
layer as the superficial muscular aponeurotic system, leads to the posterior orbicularis fascia of the eyelid. Eun et al. [5] explained that this layer can act as a migration pathway. The galea is divided into superficial and deep layers based on the frontalis. The superficial layer passes through the anterior sheath of the frontalis and orbicularis oculi muscle (OOM) and proceeds to the upper eyelid. The deep layer passes through the posterior sheath of the OOM and into the preseptal fat of the eyebrow [6]. This deep layer goes down to the posterior orbicularis fascia (Fig. 6). The injected filler or fat can migrate to the preseptal fat area along this deep layer. If an orbital septum is injured by upper blepharoplasty, the filler or fat may migrate to the preaponeurotic area. Therefore, it is recommended to avoid contact with the deep layer by injecting into the subcutaneous layer or the deeper dermis layer during injection $[5,6]$. In this patient's case, an encapsulated mass was observed in the preseptal fat area above the orbital septum (Fig. 3).

In addition to filler, injected autologous fat has also been reported to migrate from the forehead to an eyelid in the same way, causing lipogranuloma in several cases [1,7-9]. This has mainly been observed in the preseptal area or the preaponeurotic fat area, rarely in the same layer as the postseptal area. The symptoms ranged from 2 to 8 months, and surgical excision or oral steroid therapy was performed $[1,7]$. In these cases, fat was injected accidentally into the upper eyelid due to a multiple tunneling process using a blunt cannula before fat injection, and fat inoculation could be observed in the preseptal or preaponeurotic fat areas [7]. In another report, ocular swelling after a forehead fat graft was considered to be a possible cause of an oily filler leakage through the supraorbital foramen and weakening of the orbicularis retaining ligament (ORL) [8]. The supraorbital foramen is a structure near the supraorbital rim, and fat may leak into the periocular area when the structure near the foramen is weakened or widened. There is a vertical course between the superficial forehead compartment and the supraorbital rim, with the supraorbital and supratrochlear neurovasculature limiting dispersal between the forehead and supraorbital rim [9]. If this neurovascular structure is damaged, migration is possible.

The ORL is a circular structure attached to the superior orbital rim 2 to $3 \mathrm{~mm}$ above the periosteum that attaches the OOM to the orbital rim [8]. The ORL fixes the OOM to the orbital rim and protects the ocular globe. Usually it acts as a barrier to prevent the spread of infection, but injury of the ligament during surgery, including filler injection, or iatrogenic swelling can weaken this structure and allow free an oily filler to leak into the orbit [10].

To prevent this complication, an appropriate filler must be injected into the appropriate layer. Patients' desires, requirements for reversibility, tolerance of downtime, age, and skin thickness affect the selection of the filler that is most suitable for each patient. For filler injections, it is preferable is to find the method that best suits the problem at hand. A combination of abundant diagnostic tech- niques and an in-depth understanding of the characteristics of the available filler materials will lead to successful treatment results [11]. Any kind of filler can undergo migration. However, complications can be minimized if the injection is made using appropriate filler products and appropriate layers. For medium-depth fine lines and creases such as the forehead and eyebrows, hyaluronic acid filler products can achieve excellent results. Hyaluronic acid is less migratory after injection and is reversible using an antidote. The filler is placed just beneath the dermis or subcutaneous tissue to provide long-lasting and predictable results [11,12]. In case the filler may be inadvertently injected deeper than the subcutaneous layer, the author recommends using highly diluted filler [12]. We should note the reports of fatal embolic phenomena after the filler was injected into the superficial temporal artery, the supraorbital artery, and the supratrochlear artery [12].

In conclusion, the tissue layer created by the galea and posterior orbicularis fascia can act as a migration pathway. Additionally, damage to protective membranes such as the ORL or leakage to the supraorbital foramen may be migration pathways. Migration may also occur when the filler is injected deeper than the deep dermis or subcutaneous layer. In addition, migration of the filler is possible due to the continuous movement of the frontalis, or as a result of the gravity that it experiences. To prevent this complication, a licensed physician must be trained to inject the filler at the proper target layer.

\section{PATIENT CONSENT}

Patients provided written consent for the use of their images.

\section{REFERENCES}

1. Pao SI, Lin SM, Chang YH, et al. Upper eyelid granuloma: a rare delayed-onset complication secondary to cosmetic filler injection on forehead. Int Med Case Rep J 2016;9:155-7.

2. Weinberg MJ, Solish N. Complications of hyaluronic acid fillers. Facial Plast Surg 2009;25:324-8.

3. Lee JH, Choi HJ. Rare complication of silicone fluid injection presenting as multiple calcification and skin defect in both legs: a case report. Int J Low Extrem Wounds 2015;14:95-7.

4. Jordan DR, Stoica B. Filler migration: a number of mechanisms to consider. Ophthal Plast Reconstr Surg 2015;31:257-62.

5. Eun YS, Cho SH, Lee JD, et al. Periorbital lipogranuloma related to filler migration: a rare complication of facial fillers. J Cosmet Laser Ther 2014;16:149-50.

6. Tan KS, Oh SR, Priel A, et al. Surgical anatomy of the forehead, eyelids, and midface for the aesthetic surgeon. In: Massry GG, Murphy MR, Azizzadeh B, editors. Master techniques in blepharoplasty and periorbital rejuvenation. New York, NY: Springer; 2011. p. 11-24.

7. Sa HS, Woo KI, Suh YL, et al. Periorbital lipogranuloma: a previously 
unknown complication of autologous fat injections for facial augmentation. Br J Ophthalmol 2011;95:1259-63.

8. Park JW. Ocular swelling after forehead fat graft. Arch Aesthetic Plast Surg 2014;20:85-91.

9. Cotofana S, Mian A, Sykes JM, et al. An update on the anatomy of the forehead compartments. Plast Reconstr Surg 2017;139:864e-72e.

10. Ghavami A, Pessa JE, Janis J, et al. The orbicularis retaining ligament of the medial orbit: closing the circle. Plast Reconstr Surg 2008;121: 994-1001.

11. Dayan SH, Bassichis BA. Facial dermal fillers: selection of appropriate products and techniques. Aesthet Surg J 2008;28:335-47.

12. Scheuer JF, 3rd, Sieber DA, Pezeshk RA, et al. Anatomy of the facial danger zones: maximizing safety during soft-tissue filler injections. Plast Reconstr Surg 2017;139:50e-8e. 